\title{
Hemodynamic Simulation for an Anatomically Realistic Portal System
}

\author{
Harvey $\mathrm{Ho}^{1}$, Adam Bartlett ${ }^{2}$, and Peter Hunter ${ }^{1}$ \\ 1 Bioengineering Institute, University of Auckland, New Zealand \\ \{harvey.ho,p.hunter\}@auckland.ac.nz \\ 2 Department of Surgery, University of Auckland, Auckland, New Zealand \\ a.bartlett@auckland.ac.nz
}

\begin{abstract}
In this paper we present a computer model that simulates blood flow in the portal system, the vascular network that delivers nutrients and hormones to the liver from other digestion organs. Firstly the vascular geometry of a patient-specific portal system was digitised from a 3D CT image. Then blood flow in this system was solved using a set of 1D partial differential equations coupled with a bifurcation model. Some preliminary results are presented and compared with published ultrasonography and phase contrast MRA data. We further simulate a surgical procedure (portacaval shunting) that connects the portal vein with the inferior vena cava (IVC). The simulation confirms that the high pressure gradient between the portal vein and IVC leads to substantially reduced portal perfusion or even reversed flow in the portal veins, thus makes the shunting graft a flow 'highway' in directing portal flow.
\end{abstract}

\section{Introduction}

The portal system is the venous network that starts from the intestine and terminates at capillaries in the liver [2]. This network, together with the splanchnic network which are the veins connecting the spleen and the liver, constitutes a vital pathway for digestion. It delivers nutrients from the intestine and hormones from the pancreas and spleen into the liver, where nutrient metabolism and detoxification further take place. Pathology of the portal system will hamper food digestion and may even cause death. For example, liver cirrhosis leads to an increased resistance to portal flow, and in turn causes portal hypertension. Serious portal hypertension is the major cause of bleeding esophagus varices, which is fatal if not treated urgently 2.

Hemodynamics research of the portal system is the study of blood flow in the system, in both healthy and pathological conditions. The importance of this research can be appreciated from the amount of literature on this subject over the last several decades. Quantitative flow data have been collected from in vivo (e.g., in [134]) and in vitro measurements (e.g., in [5]). Computer models have been designed in line with in vitro experiments and clinical observations to explain portal circulation phenomena (e.g., in [5]). These models have provided great insights into the physiology of portal and splanchnic circulation, thus aided 
clinical and physiological research. For example, an analog electronic model was proposed to provide the theoretical basis for a portacaval shunting procedure [5]. These hemodynamic models, however, are mostly zero-dimensional (0D) models that lump the blood vessels into electronic components (e.g., resistors, capacitors) to represent their resistance and compliance.

The advance of medical scanning techniques and increasing computational power have made it possible to study the portal system in great detail. Not only can a large portal network be segmented for structural analysis [6], but the blood flow in a local portal bifurcation can be simulated for functional study [7]. However, blood flow modelling for a large image-based portal/splanchnic network has not yet been reported, to our knowledge.

The purpose of this paper is two fold. First, we present a vascular network construction method that facilitates further flow simulation. Second, we model blood flow in the portal system by solving governing flow equations. Based on this model, we simulate a surgical procedure whereby a shunting graft is employed to connect the portal vein and the inferior vena cava (IVC). We present some preliminary results and compare the results with published in vivo data. We also comment on the performance of this model in the Discussion section.

\section{Method}

\subsection{Blood Vessel Digitisation and Medical Imaging}

We retrospectively studied a 3D CT image of an anonymous patient. The image spanned the whole torso (Fig. 1(a)) and its voxel resolution was $0.879 \mathrm{~mm} \times$ $0.879 \mathrm{~mm} \times 0.625 \mathrm{~mm}$. The scanning was performed at the portal-venous phase to enhance the portal and hepatic veins (indicated by arrows) via a contrast agent. The objective of this work was to simulate blood flow in these vessels by solving flow equations. To this end we needed to identify the topology of the network, clarify the connectivity between parent/daughter vessels, and determine the radius and length of each blood vessel in the network. We employed an imaging and visualization tool CMGUI (http://www.cmiss.org/cmgui) for this work. The procedure was as follows:

1. Locate the key points (nodes) along the centreline of blood vessels;

2. Measure vessel diameter at each node; and

3. Connect these nodes using one dimensional (1D) finite element elements.

This process is illustrated in Fig. 1(b): the small red dots are the key nodes, which are connected by 1D linear elements, then updated to incorporate vessel radius to mimic blood vessels. The final portal network, plus the IVC, the renal and iliac veins (as part of systemic veins), are shown in Fig. 1(c). The portal veins are colored in gold, and the systemic veins in blue. We also digitised the torso, liver, heart and the spleen as spatial references for these blood vessels. The timeframe for the digitisation process was 15 hours for the portal vasculature, and 16 hours for the organs and the torso. The whole vasculature contains 109 venous segments, with 57 branch (bifurcating or merging) points, 42 inlets and 18 outlets. 
(a)

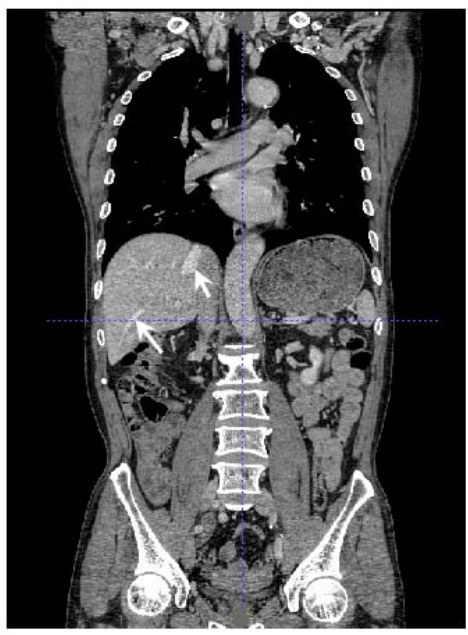

(c)
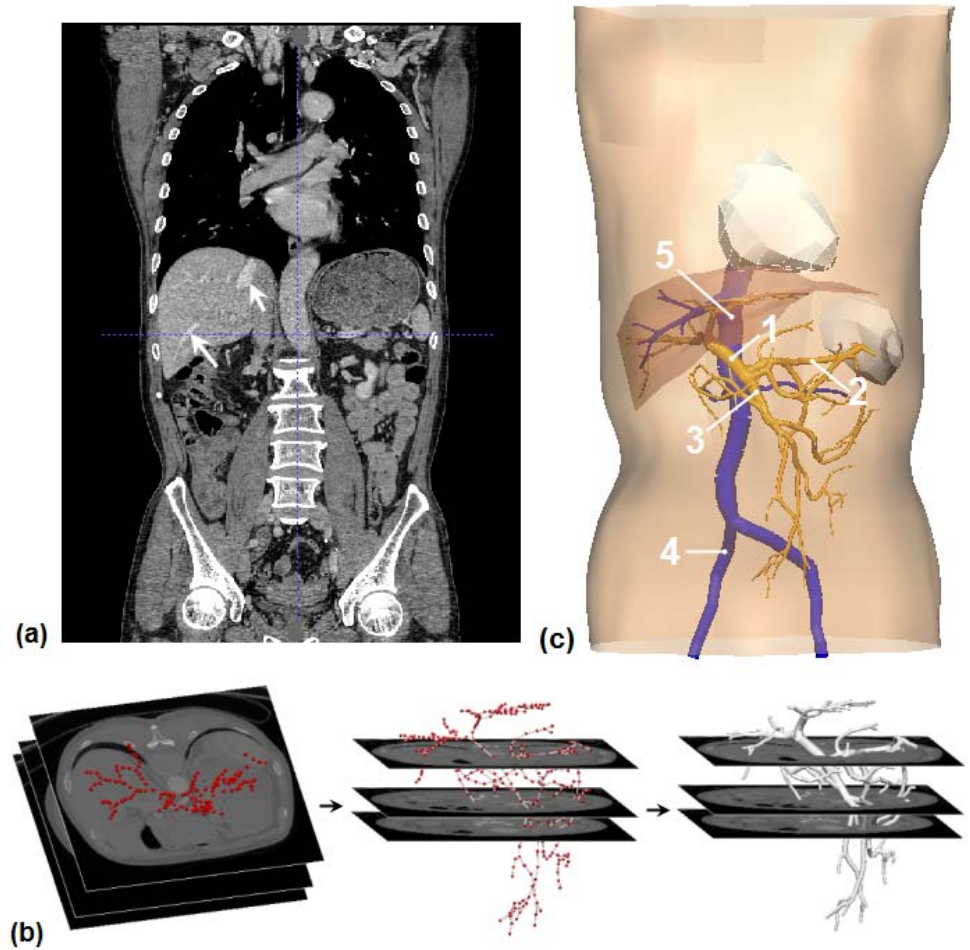

Fig. 1. (a) CT image: the arrows indicate hepatic veins; (b) The process of vessel digitisation; (c) Digitised portal veins and systemic veins. 1 - portal vein; 2 - splenic vein; 3 - superior mesenteric vein (SMV); 4 -iliac vein; 5 - inferior vena cava (IVC).

\subsection{Haemodynamics Modelling}

We adopted a 1D flow solver to simulate flow in this vasculature. A 1D model essentially works by regarding the longitudinal (or axial) direction as the major flow direction, ignoring flow in other directions [8]. The governing equations are:

$$
\begin{gathered}
\frac{\partial R}{\partial t}+V \frac{\partial R}{\partial x}+\frac{R}{2} \frac{\partial V}{\partial x}=0 \\
\frac{\partial V}{\partial t}+(2 \alpha-1) V \frac{\partial V}{\partial x}+2(\alpha-1) \frac{V^{2}}{R} \frac{\partial R}{\partial x}+\frac{1}{\rho} \frac{\partial P}{\partial x}=-2 \frac{v \alpha}{\alpha-1} \frac{V}{R^{2}} \\
P=\frac{2}{3} \frac{E h_{0}}{R_{0}}\left(\frac{R^{2}}{R_{0}^{2}}-1\right)
\end{gathered}
$$

where $R, P, V$ are the vessel radius, pressure and flow velocity, respectively. Eqs. (1) and (2) are mass and momentum conservation equations. Eq. (3) is an 
empirical vessel wall equation that describes the relationship between transmural pressure and vessel radius. $E, h_{0}, R_{0}$ are Young's modulus, wall thickness and unstressed radius, respectively. $\alpha$ is a constant that defines the axial flow profile. Note, that Eq. (3) only describes the scenario where a vein is expanded (i.e., $P>0$ ) and ignores its (partly) collapsed status. The later case is not considered in this work for modelling simplicity's reason.

Eqs. (1)-(3) constitute a closed partial differential equation system for solving hemodynamics in a single vessel. We employed a second-order MacCormack finite difference scheme to solve this system. The spatial and temporal steps were set as $1 \mathrm{~mm}$ and 0.1 milliseconds respectively for numerical convergence. To simulate flow in a network, we incorporated a bifurcation model which evaluates the flow distribution, velocity and pressure gradient across branches, and therefore the whole tree. We refer the interested reader to the literature 8] for more mathematical details of the bifurcation model.

\subsection{Simulation of Portacaval Shunting}

Portacaval shunting is a surgical procedure that connects the portal vein with the IVC using an H-graft 135. The rationale is that the blood pressure in the IVC is lower than that in the portal vein. The pressure gradient drains portal blood flow into IVC via the shunt to reduce portal pressure and hence relieve the lethal threat of variceal bleeding 2 . The drawback of this procedure, however, is that it can lead to hepatic encephalopathy (i.e., mind-confusion or coma). This is because a large portion of portal blood bypasses the liver, where detoxification takes place, and flows into the brain via blood circulation [2].

The effectiveness of this procedure was investigated by various surgical groups, e.g., by Rypins et. al. 15. They found that different shunt sizes led to different clinical outcome. They also used a mathematical model to justify their observations [5]. Based on the pressure and flow data in [5], we can construct a virtual shunt to simulate this procedure, utilizing the vascular network of Fig. 1. In particular, we are able to analyze the portal flow diversion due to the shunting, as shown in the next section.

\section{Results}

\subsection{Flow Simulation for the Portal System}

In the first step we simulate flow in the portal system without considering the systemic veins and the shunt. Since the actual measurements for the inlets and outlets of the portal system were not available, we took an empirical approach in the boundary condition (BC) arrangement. This approach is illustrated in Fig. 2. The red dots represent different groups of inlets or outlets that shared the same pressure BC intra-group but there was a pressure gradient between these groups, thus drove flow in the tree. The pressure data in Fig. 2 were adopted from [5]. 

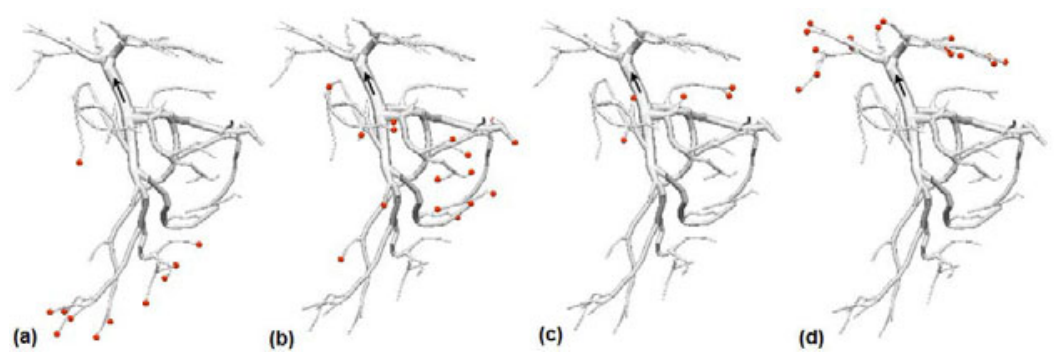

Fig. 2. An empirical approach to pressure boundary condition configuration: (a) inlet group 1: $\mathrm{P} 1=22.5 \mathrm{mmHg}$; (b) inlet group 2: $\mathrm{P} 2=20.6 \mathrm{mmHg}$; (c) inlet group 3: $\mathrm{P} 3=18.75 \mathrm{mmHg}$; (4) outlet group: $\mathrm{P} 4=15 \mathrm{mmHg}$. Note: $1 \mathrm{kPa} \approx 7.5 \mathrm{mmHg}$.

Table 1. Comparison of flow velocities in major veins (Unit: $\mathrm{mm} / \mathrm{s}$ )

\begin{tabular}{c|c|c|c}
\hline Name & Our model & Koslin et al [4] & George [7] \\
\hline Portal vein & 71 & 65 & $79.9 \pm 37.0$ \\
SMV & 65.4 & - & $54.4 \pm 24.5$ \\
Splenic vein & 33.6 & - & $88.5 \pm 19.5$ \\
\hline
\end{tabular}

As the initial BC, the pressure across the tree was assumed to be the same and there was no flow in the network. Then the blood pressures at the inlets were raised gradually within 0.5 seconds until they reached their respective configured values (as per Fig. 2). The inlet pressures were then held unchanged for another 0.5 seconds to stabilize the simulation. The whole computation took 105 seconds to complete on a desktop computer (Intel Core II, 2.4GHz CPU, 2GB RAM).

Fig. 3(a) and (b) show the pressure and velocity distribution across the portal network. The hierarchical pressure gradient between the inlets and outlets can be appreciated from Fig. 3(a). The pressure profile indicates the time course of inlet pressure BC. The flow velocity distribution, as shown in Fig. 3(b), reflects the fact that the flow velocity is strongly related to vessel diameters. For example, blood flow will be accelerated in a tapering vessel, but decelerated in an expanding vessel.

A comparison was made between the flow velocities calculated from the model and that measured using Duplex ultrasonic measurement 4] and phase contrast MRA 7]. The comparison result, shown in Table 1 , indicates that the flow velocities in the portal vein and SMV of our model are consistent with that in literature. The discrepancy of flow velocities in the splenic vein may however be minimized by using a different set of $\mathrm{BC}$ and requires further investigation.

\subsection{Portacaval Shunt Simulation}

Based on the above model, we added systemic veins and the portacaval shunting into the simulation. We analyzed two flow cases: Case 1, an 8mm graft was 

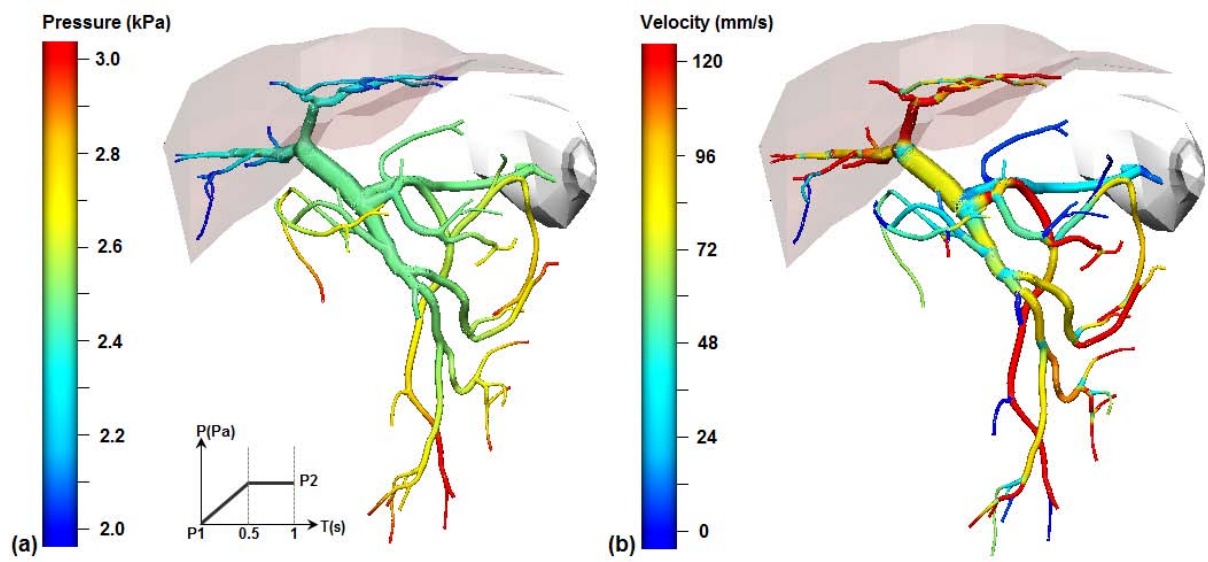

Fig. 3. Blood flow simulation in the portal system: (a) pressure distribution. The pressure profile is the time course of an inlet pressure: it increases from P1 to P2 within 0.5 seconds, then held steady for another 0.5 seconds. The outlet pressure stays at P1 - the pressure gradient between the inlets and outlets drives blood flow; (b) flow velocity distribution: the flow velocity at the portal vein is $71 \mathrm{~mm} / \mathrm{s}$.

Table 2. Flow distribution in the portal vein and the shunt (Unit: $\mathrm{ml} / \mathrm{s}$ )

\begin{tabular}{c|c|c}
\hline Venous segment & Case 1 & Case 2 \\
\hline 1 & 14.46 & 48.51 \\
2 & 19.74 & 31.19 \\
3 & 5.35 & 17.73 \\
\hline
\end{tabular}

employed and a prograde flow in the portal vein was sustained; and Case 2, a $10 \mathrm{~mm}$ shunt was used and the flow in the portal vein was reversed. Both scenarios were observed clinically, e.g., as reported in [1] and [3].

Fig. 4 shows the computational results in the two cases. The flow directions in the shunt, the portal vein pre- and aft-shunt (marked 1, 2, and 3) are indicated by arrows in the sketches. The flow rates $\left(F=V \cdot \pi R^{2}\right)$ in these segments are shown in Table 2. In Case $1, F_{1}+F_{3}=19.81(\mathrm{ml} / \mathrm{s}) \approx F_{2}$; in Case 2, $F_{2}+F_{3}=48.92(\mathrm{ml} / \mathrm{s}) \approx F_{1}$. Thus mass conservation in the shunting branch was obeyed.

We observed that a large portion $(14.46 \div 19.74 \approx 73 \%)$ of portal blood flowed to the shunt in Case 1. While this helps to decompress the portal hypertension, the substantially reduced portal perfusion implies that toxins in the blood were not removed sufficiently. In Case 2 the prograde and retrograde portal merged into shunting flow, thus causing a high flow rate in the graft. Again, this can lead to a high rate of hepatic encephalopathy 11. The mechanism underlying these phenomena is that the high pressure gradient between the portal vein and the IVC makes the shunting graft a flow 'highway' in directing portal flow. 


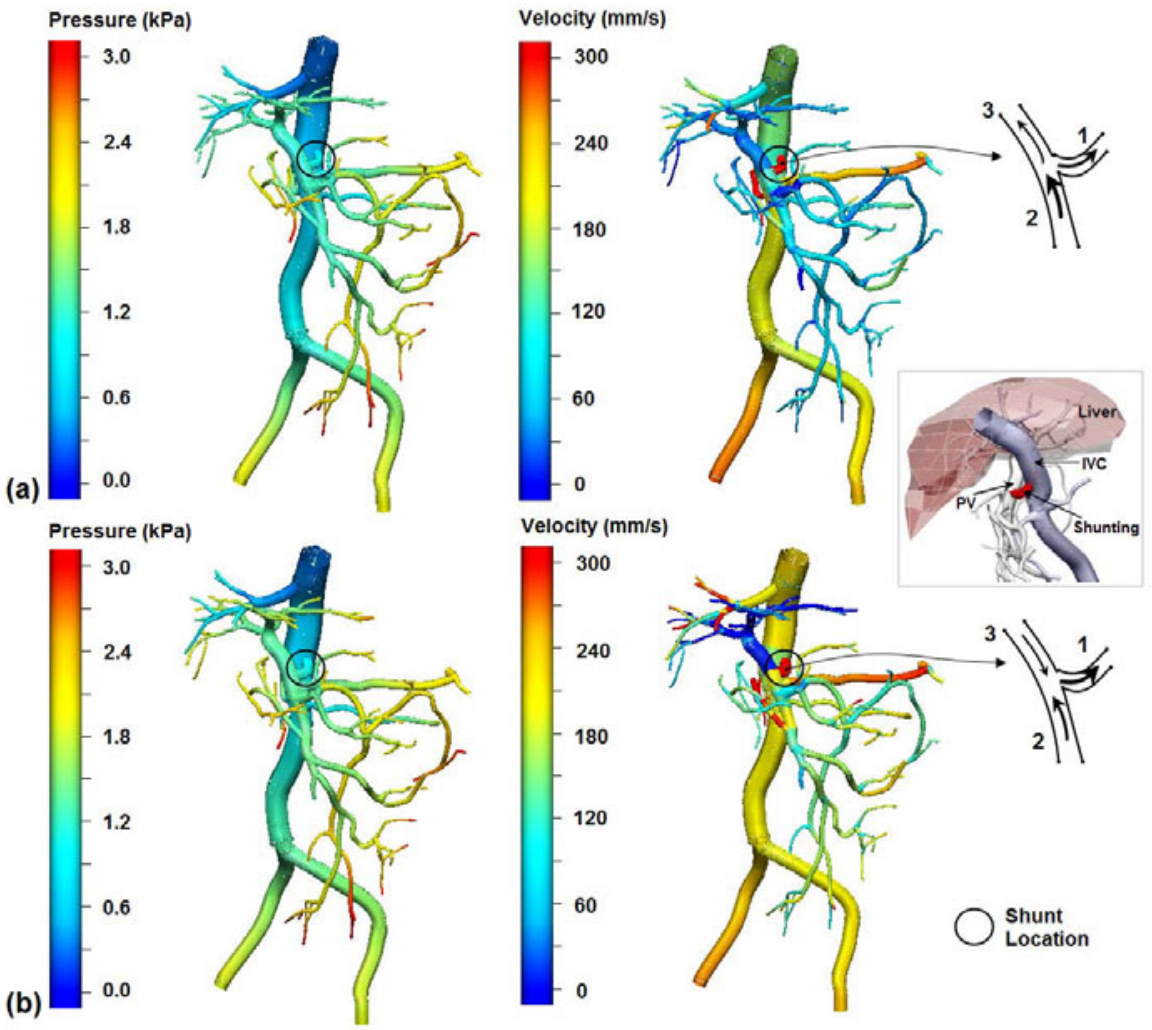

Fig. 4. Flow simulation for the portalcaval shunt: (a) Case 1: prograde flow in the portal vein (using a $8 \mathrm{~mm}$ graft); (b) Case 2: retrograde flow in the portal vein (using a $10 \mathrm{~mm}$ graft). The sketches show the flow directions in the portal vein and the shunt.

\section{Discussion}

This work represents the first portal flow model built on an anatomically accurate portal/splanchnic tree digitised from a 3D scanning image. We employed a 1D solver to calculate the blood flow in this vasculature and used the model to simulate hemodynamics after a surgical procedure that connects the portal vein with the IVC. Some preliminary results were presented and compared with in vivo measurements and clinical observations.

The reason we adopted a $1 \mathrm{D}$ model instead of a 3D model for the vascular network was due to its computational efficiency. Some other advantages of the 1D model are its ability to capture pressure wave [8] and its flexibility in modelling wall elasticity. However, the convergence of a $1 \mathrm{D}$ model is largely affected by boundary conditions. This is particularly true for a large vasculature such as the 
one modelled in this paper. While the hierarchical pressure boundary scheme employed in this work can generate a pressure gradient in the network thus the flow, future measured in vivo data can further improve the model.

One of the unique flow characteristics of portal circulation is that the hepatic resistance to portal flow is very low. For example, only a $0.5 \mathrm{mmHg}$ pressure gradient is required for portal perfusion [5]. Therefore, a small variation of portal pressure due to, e.g., liver cirrhosis, can disturb the portal circulation. These interwoven physiological phenomena require adoption of a combination of flow models, from 0D to 3D, to yield an integrative picture, and thus better interpret and combat portal system diseases.

\section{Conclusion}

In this paper we simulated blood flow in a portal system that was digitised from a 3D CT image. We used this model to simulate a surgical procedure that connects the portal vein and the IVC via a virtual shunting graft. The simulation confirms the clinical observations that the shunting can induce a substantially reduced portal perfusion or a reversed flow in the portal veins.

\section{References}

1. Sarfeh, I.J., Rypins, E.B., Mason, G.R.: A systematic appraisal of portacaval Hgraft diameters: Clinical and hemodynamic perspectives. Annals of Surgery 204(4), 356-363 (1986)

2. Boyer, T.D., Wright, T.L., Manns, M.P.: Zakim and Boyer's Hepatology: A textbook of Liver Disease, 5th edn. Saunders (2006)

3. Lafortune, M., Patriquin, H., Pomier, G., Huet, P., Weber, A., Lavoie, P., Blanchard, H., Breton, G.: Hemodynamic changes in portal circulation after portosystemic shunts: use of duplex sonography in 43 patients. Am. J. Roentgenol. 149(4), 701-706 (1987)

4. Koslin, D.B., Berland, L.L.: Duplex Doppler examination of the liver and portal venous system. Journal of Clinical Ultrasound 15(9), 675-686 (1987)

5. Rypins, E.B., Rosenberg, K.M., Sarfeh, I., Houck, J., Conroy, R.M., Milne, N.: Computer analysis of portal hemodynamics after small-diameter portacaval H- grafts: The theoretical basis for partial shunting. Journal of Surgical Research 42(4), 354361 (1987)

6. Selle, D., Preim, B., Schenk, A., Peitgen, H.: Analysis of vasculature for liver surgical planning. IEEE Transactions on Medical Imaging 21(11), 1344-1357 (2002)

7. George, S.: Hemodynamic investigation of the liver using magnetic resonance imaging and computational fluid dynamics. PhD thesis. Georgia Institute of Technology (2008)

8. Smith, N.P., Pullan, A.J., Hunter, P.J.: An anatomically based model of transient coronary blood flow in the heart. SIAM Journal on Applied Mathematics 62(3), 990-1018 (2001) 